\title{
c-Jun-dependent inhibition of cutaneous procollagen transcription following ultraviolet irradiation is reversed by all-trans retinoic acid
}

\author{
Gary J. Fisher, Subhash Datta, ZengQuan Wang, Xiao-Yan Li, Taihao Quan, \\ Jin Ho Chung, Sewon Kang, and John J. Voorhees \\ Department of Dermatology, University of Michigan, Ann Arbor, Michigan, USA \\ Address correspondence to: Gary J. Fisher, Department of Dermatology, 1150 West Medical Center Drive, \\ Medical Science I, Room 6447, Ann Arbor, Michigan 48109-0609, USA. \\ Phone: (734) 763-1469; Fax: (734) 647-0076; E-mail: dianemch@umich.edu.
}

Received for publication January 11, 2000, and accepted in revised form July 28, 2000.

\begin{abstract}
The aged appearance of skin following repeated exposure to solar ultraviolet (UV) irradiation stems largely from damage to cutaneous connective tissue, which is composed primarily of type I and type III collagens. We report here that a single exposure to UV irradiation causes significant loss of procollagen synthesis in human skin. Expression of type I and type III procollagens is substantially reduced within 24 hours after a single UV exposure, even at UV doses that cause only minimal skin reddening. Daily UV exposures over 4 days result in sustained reductions of both type I and type III procollagen protein levels for at least 24 hours after the final UV exposure. UV inhibition of type I procollagen synthesis is mediated in part by c-Jun, which is induced by UV irradiation and interferes with procollagen transcription. Pretreatment of human skin in vivo with all-trans retinoic acid inhibits UV induction of c-Jun and protects skin against loss of procollagen synthesis. We have reported previously that UV irradiation induces matrix-degrading metalloproteinases in human skin and that pretreatment of skin with all-trans retinoic acid inhibits this induction. UV irradiation, therefore, damages human skin connective tissue by simultaneously inhibiting procollagen synthesis and stimulating collagen breakdown. All-trans retinoic acid protects against both of these deleterious effects and may thereby retard premature skin aging.
\end{abstract}

J. Clin. Invest. 106:663-670 (2000).

\section{Introduction}

Solar ultraviolet (UV) irradiation damages human skin and causes premature skin aging (photoaging) characterized by thickening, rough texture, coarse wrinkles, and mottled pigmentation (1). Histologic and ultrastructural studies have revealed that the major alterations in photoaged skin are localized in the connective tissue (dermis), which is composed predominantly of type I and type III collagen, elastin, proteoglycans, and fibronectin. Damage induced by UV irradiation is manifested primarily as the disorganization of collagen fibrils (2) that constitute the bulk ( $90 \%$ dry weight) of skin connective tissue and accumulation of abnormal, amorphous, elastin-containing material (3). Since collagen fibrils and elastin are responsible for the strength and resiliency of skin (4), their disarrangement with photoaging causes skin to appear aged.

Biochemical evidence of connective tissue alterations in photoaged human skin includes reduced levels of types I and III collagen precursors $(5,6)$ and cross-links (7), increased ratio of type III to type I collagen (6), and increased levels of elastin (8). Additionally, wrinkle reduction in photodamaged human and mouse skin, after treatment with topical all-trans retinoic acid, correlates with increased dermal procollagen synthesis (9-11).
Fibroblasts that reside within skin connective tissue synthesize and secrete type I and type III procollagens. Type I procollagen typically is composed of two $\alpha 1$ chains and one $\alpha 2$ chain, although homotrimers of $\alpha 1$ chains have been described in normal skin and certain diseases $(12,13)$. Type III procollagen is composed of three identical $\alpha 1$ chains (distinct from type I $\alpha 1$ chains). Type I and III procollagens contain globular amino- and carboxy-terminal domains, which make these proteins soluble. After secretion of type I and type III procollagen, their amino- and carboxy-terminal domains are cleaved by specific proteases $(14,15)$, resulting in formation of mature collagen, which spontaneously assembles into thin collagen fibrils. Because type I and type III procollagens and their partially processed forms are precursor molecules of mature collagen, their levels generally reflect the level of collagen biosynthesis $(16,17)$.

Mature type I collagen in skin undergoes continuous turnover, which is required for optimal connective-tissue function (18). Collagen turnover is regulated by both its rate of synthesis and its rate of breakdown. In mammals, breakdown of collagen fibrils is dependent on the action of one of three known collagenases, MMP-1, MMP-8, or MMP-13, which initiates collagen cleavage at one specific site. Imbalance in collagen syn- 
thesis and degradation can result in thickened, hardened skin due to collagen build up, or thin, fragile skin, due to collagen deficiency.

We have reported previously that low-dose UV irradiation induces collagenase and other matrix metalloproteinases that degrade mature collagen in human skin in vivo $(19,20)$. Since connective-tissue remodeling involves synthesis as well as degradation, we investigated the effect of UV irradiation on regulation of procollagen synthesis in human skin in vivo.

\section{Methods}

UV irradiation and tissue procurement. Healthy adult Caucasian subjects (45 subjects, approximately equal numbers of males and females), with light to mild skin pigmentation, were irradiated with four F36T12 ERE-VHO UVB tubes (UVB/UVA2 source), as described $(20,21)$. A Kodacel TA401/407 filter was mounted $4 \mathrm{~cm}$ in front of the tubes to remove wavelengths below $290 \mathrm{~nm}$ (UVC), since UVC does not reach Earth's surface. Irradiation intensity was monitored using an IL1400A phototherapy radiometer and an SED240/UVB-1/W photodetector (International Light, Newburyport, Massachusetts, USA). Spectroradiometry was performed using an Optronic Laboratories (Orlando, Florida, USA) OL 754 system. For some studies, subjects were irradiated with a xenon arc solar simulator (Spex Instruments S.A. Inc., Edison, New Jersey, USA). Minimal erythema dose (MED) for each subject was determined 24 hours after irradiation. Irradiated and nonirradiated skin samples were obtained from each subject by punch biopsy, as described previously (22). All-trans retinoic acid (0.1\%) and its vehicle (70\% ethanol, 30\% propylene/glycol, $0.05 \%$ butylated hydroxytoluene) were applied to skin under light-tight occlusion, 8 or 48 hours before UV treatment. For studies with multiple time points or treatments, tissue was obtained from each subject for each time point or treatment. $n$ represents the number of subjects from whom tissue was taken for analysis. All procedures involving human subjects were approved by the University of Michigan Institutional Review Board, and all subjects provided written informed consent.

Western analysis. Type $\mathrm{I}(\alpha 1)$ procollagen and type $\mathrm{III}(\alpha 1)$ procollagen were measured in skin extracts by Western analysis (Ab's from Research Diagnostics Inc., Flanders, New Jersey, USA) (23). C-Jun protein (Ab from Transduction Laboratories, Lexington, Kentucky, USA) was detected in nuclear extracts from cultured adult human skin fibroblasts using Western analysis (5). Immunoreactive proteins were visualized by enhanced chemiluminescence detection and quantified by laser densitometry or visualized by enhanced chemifluorescence detection and quantified by STORM PhosphorImager (Molecular Dynamics, Sunnyvale, California, USA).

Northern analysis. Frozen skin samples were ground with mortar and pestle under liquid nitrogen. Total RNA was isolated and hybridized against [ $\left.{ }^{32} \mathrm{P}\right]$-labeled type $\mathrm{I}(\alpha 1)$, type $\mathrm{I}(\alpha 2)$, type $\mathrm{III}(\alpha 1)$ procollagen, and $\left[{ }^{32} \mathrm{P}\right] 36 \mathrm{~B} 4 \mathrm{cDNA}$ probes, as described previously (24). Hybridization signals were quantified by STORM PhosphorImager (Molecular Dynamics). Hybridization of 36B4 was used as an internal control to normalize all results.

In situ bybridization. Digoxigenin-labeled sense and antisense riboprobes for human type $\mathrm{I}(\alpha 1)$ and type III $(\alpha 1)$ procollagen mRNA were synthesized using T3 and $\mathrm{T} 7$ ribonucleic acid polymerases. Frozen skin sections $(5 \mu \mathrm{m})$ were mounted, fixed, treated, and hybridized, as described previously (24). Hybridization signals were detected immunohistochemically using alkaline phosphatase-conjugated antidigoxigenin Ab.

Transfection of fibroblasts and CAT assay. Primary cultured adult human skin fibroblasts (25) were transfected with $2 \mu \mathrm{g}$ COL1A2 reporter-gene plasmid (-772 to +58) (26) alone or together with $100 \mathrm{ng}$ expression vectors for either wild-type or dominant-negative mutant c-Jun (TAM-67) (27) using FuGene6 (Boehringer Mannheim, Indianapolis, Indiana, USA). All cells were cotransfected with pXJ40-LacZ plasmid $(2 \mu \mathrm{g})$, containing the $\beta$-galactosidase gene. Twenty-four hours after transfection, cells were washed, covered with $0.5 \mathrm{~mL}$ PBS, and either irradiated with Kodacel-filtered UVB tubes, as described $\left(30 \mathrm{~mJ} / \mathrm{cm}^{2}\right)$, or sham irradiated. Cells were harvested 8 hours after irradiation, lysates were normalized to $\beta$-galactosidase activity, and chloramphenicol acetyl transferase (CAT) activity measured as described (28).

Statistics. Endpoints involving single comparisons were analyzed with the paired $t$ test. Endpoints involving multiple treatments or time intervals were analyzed with a repeated measures ANOVA. Specific comparisons were formed with either Tukey's Studentized range test for all pairwise comparisons or Dunnett's $t$ test for treatmentversus-control comparisons. Both tests control the experiment-wise type I error rate at the stated alpha level of 0.05 . Summary data are represented as means plus or minus one SEM. The data were analyzed with SAS statistical software (SAS Institute Inc., Cary, North Carolina, USA).

\section{Results}

Acute UV transiently reduces type I and III procollagen $m R N A$ and protein levels in human skin in vivo. Initially, we determined the effect of a single UV exposure (2 MED) on type I and type III procollagen mRNA expression in human skin in vivo. Figure $1, a-j$, shows representative photomicrographs that display the epidermis and adjacent upper portion of skin connective tissue (dermis). Riboprobe in situ hybridization revealed that numerous cells in skin connective tissue expressed mRNA for type I $(\alpha 1)$ (Figure 1a) and type III $(\alpha 1)$ (Figure 1f) procollagen in sun-protected, nonirradiated human skin. Within 8 hours after a single UV exposure, cellular expression of both type $\mathrm{I}(\alpha 1)$ (Figure $1 \mathrm{~b}$ ) and type III $(\alpha 1)$ procollagen mRNA (Figure $1 \mathrm{~g}$ ) were substantially reduced in the upper dermis. At 24 hours after UV exposure, neither type $\mathrm{I}(\alpha 1)$ (Figure $1 \mathrm{c}$ ) nor type $\operatorname{III}(\alpha 1)$ procollagen mRNA (Figure $1 \mathrm{~h}$ ) was detectable in the 

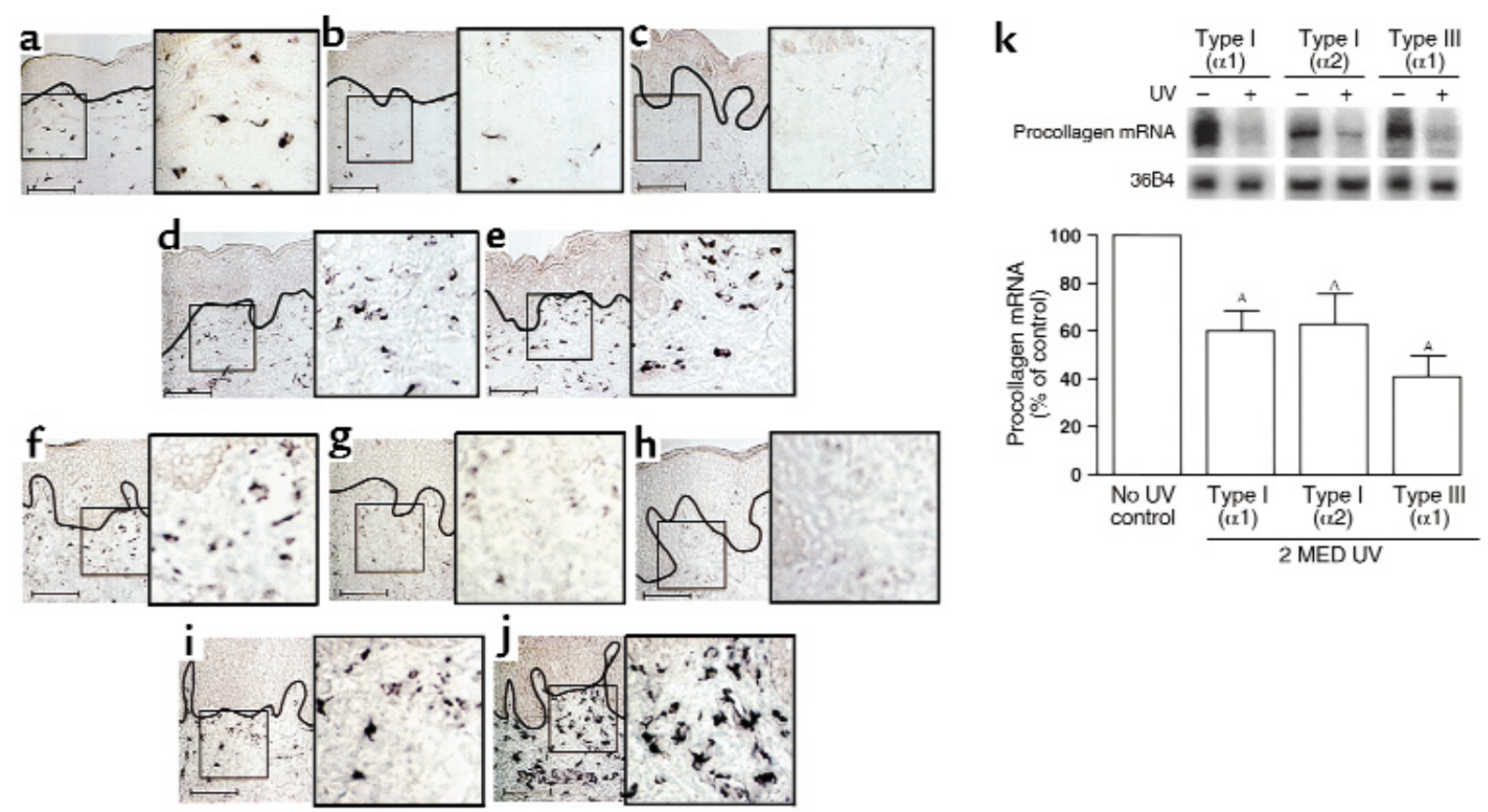

Figure 1

UV irradiation inhibits type I and type III procollagen gene expression in human skin in vivo. Nonirradiated and adjacent UV-irradiated (2 MED) human skin samples were obtained at the times indicated after UV irradiation. Skin was analyzed for type I( $\alpha 1)$ and type III( $\alpha 1)$ procollagen mRNA expression by digoxigenin-riboprobe in situ hybridization. (a-e) Type I( $\alpha 1)$ procollagen and (f-j) type III( $(\alpha 1)$ procollagen mRNA expression in human skin. $\mathbf{a}$ and $\mathbf{f}: 0, \mathbf{b}$ and $\mathbf{g}: 8, \mathbf{c}$ and $\mathbf{h}: 24, \mathbf{d}$ and $\mathbf{i}: 48$, and $\mathbf{e}$ and $\mathbf{j}: 72$ hours after irradiation. Data are representative of six subjects. Scale bar $=10 \mu \mathrm{m}$. In $\mathbf{a}-\mathbf{j}$, solid black lines demarcate border between epidermis and dermal connective tissue. Areas outlined in black are shown in 2.5-fold enlargements. (k) Northern analysis of type I( $\alpha 1)$, type I( $\alpha 2)$, and type III $(\alpha 1)$ procollagen mRNA levels in human skin. Total mRNA (20 $\mu \mathrm{g}$ /lane) was analyzed from nonirradiated and UV-irradiated ( 2 MED) human skin, obtained 24 hours after UV irradiation (2 MED), and 36B4 mRNA was used as an internal control. Inset shows representative Northern blot. Procollagen mRNA levels were normalized to 36B4 mRNA levels. $n=6-7$. ${ }^{A} P<0.01$ vs. no UV exposure.

upper dermis. During the next 24 hours (i.e., 48 hours after UV exposure) cellular expression of type $\mathrm{I}(\alpha 1)$ (Figure 1d) and type III $(\alpha 1)$ procollagen mRNA (Figure 1i) in the upper dermis returned to near their original levels (i.e., before UV exposure). Both type $\mathrm{I}(\alpha 1)$ and type III $(\alpha 1)$ procollagen mRNA expression in the upper dermis appeared to increase above their initial levels by 72 hours after UV exposure (Figure 1, e and j). Similar changes in cellular expression of type $\mathrm{I}(\alpha 2)$ procollagen mRNA after UV irradiation of human skin were observed (data not shown).

In nonirradiated skin, type I and type III procollagen transcripts were expressed in cells throughout the entire depth of skin connective tissue. However, UVinduced alterations in procollagen gene expression described above were most prominent in the upper third of the connective tissue. To quantify UV-induced reduction of procollagen gene expression, we performed Northern analysis on skin samples obtained 24 hours after UV irradiation (2 MED). UV irradiation reduced type $I(\alpha 1)$ and type $I(\alpha 2)$ procollagen mRNA approximately $40 \%$ and type III $(\alpha 1)$ procollagen mRNA approximately $60 \%$ (Figure $1 \mathrm{k}$ ).

Immunohistology revealed that alterations of type I and type III procollagen protein expression in fibrob- lasts, after a single UV exposure (2 MED), were similar to those of their mRNA expression (data not shown). Western analyses, using $A b$ 's that recognize the amino-terminal propeptide of type I $(\alpha 1)$ and type III $(\alpha 1)$ procollagen, revealed that intact type $\mathrm{I}(\alpha 1)$ and type $\mathrm{III}(\alpha 1)$ procollagen and their partially processed $\mathrm{pN}$ forms (procollagen lacking its carboxy-terminal propeptide region) were substantially reduced within 8 hours after UV exposure (Figure 2, $a$ and $b$ ). Both type $I(\alpha 1)$ procollagen (Figure $2 \mathrm{a}$ ) and type III $(\alpha 1)$ procollagen (Figure $2 \mathrm{~b}$ ) protein levels remained reduced at 24 hours after UV exposure. By 48 hours after $\mathrm{UV}$ exposure, type $\mathrm{I}(\alpha 1)$ procollagen (Figure $2 \mathrm{a}$ ) and type III $(\alpha 1)$ procollagen (Figure $2 \mathrm{~b}$ ) returned to near their original levels (i.e., before UV exposure). Alterations of type $\mathrm{I}(\alpha 1) \mathrm{pN}$ collagen (Figure $2 \mathrm{a}$ ) and type $\mathrm{III}(\alpha 1) \mathrm{pN}$ collagen (Figure $2 \mathrm{~b}$ ) after UV irradiation mirrored those of their intact procollagen precursors.

Multiple UV exposures cause sustained reductions of type I and type III procollagen protein levels in human skin in vivo. Solar damage to skin connective tissue is cumulative. We therefore determined the effect of multiple UV exposures on type I and type III procollagen protein levels. Subjects were irradiated on four separate sites, one, two, three, or four times (1 MED each), at 24-hour intervals. Skin samples were obtained 24 hours after each UV 

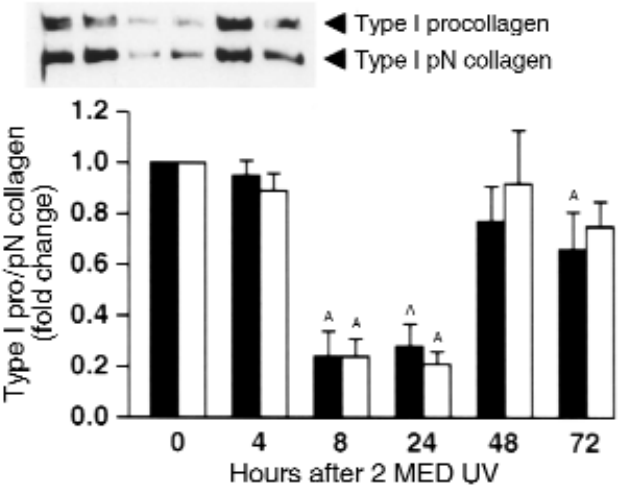

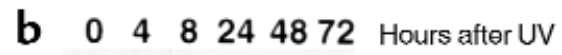
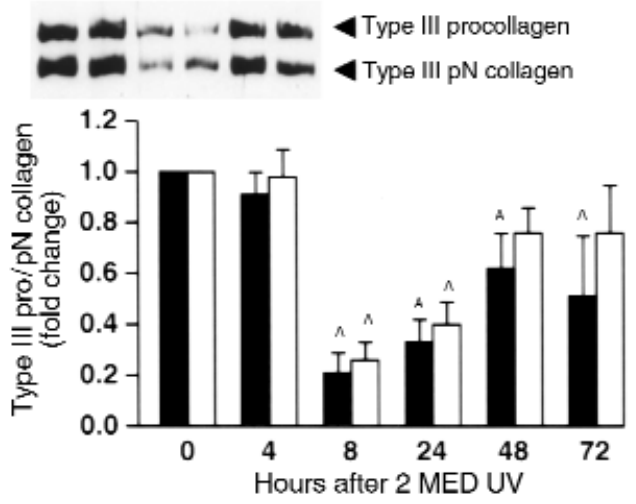

Figure 2

UV reduces type I and type III procollagen protein levels in human skin in vivo. Nonirradiated and adjacent UV-irradiated (2 MED) human skin samples were obtained at the times indicated after UV irradiation. Skin was analyzed for type I( $\alpha 1)$ and type III( $\alpha 1)$ procollagen protein levels using Western blot method. (a) Type I procollagen (filled bars) and type I pN collagen (open bars) protein levels in human skin. Inset shows representative Western blot. (b) Type III procollagen (filled bars) and type III pN collagen (open bars) protein levels in human skin. Inset shows representative Western blot. $n=7-9$ subjects. ${ }^{A} P<0.05$ vs. no UV exposure.

exposure and analyzed for type I and type III procollagen using Western blot analysis. As expected, a single UV exposure caused significant reduction of type I (Figure 3a) and type III (Figure $3 \mathrm{~b}$ ) procollagen and $\mathrm{pN}$ collagen protein levels. Additional UV exposures resulted in sustained reductions of both type I (Figure 3a) and type III (Figure $3 \mathrm{~b}$ ) procollagen and $\mathrm{pN}$ collagen levels.

$U V$ dose dependence for reduction of type I procollagen protein levels in human skin in vivo. We next determined the UV dose dependence for reduction of type I procollagen protein levels in human skin. For these studies we used two different UV sources: a UVB/UVA2 source that emitted predominantly UVB $(290-320 \mathrm{~nm}$; used in the studies described above) and a solar simulator, which emitted predominantly UVA (320-400 nm) (Figure 4a). Human skin was exposed in vivo to UV doses between $0.1 \mathrm{MED}$ and $2 \mathrm{MED}$ from each source and analyzed for type I procollagen and $\mathrm{pN}$ collagen protein levels using Western blot analysis. A dose of 0.5 MED (one-half the dose required to cause slight skin reddening) from the UVB source caused significant reduction of type I procollagen and pN collagen (Figure $4 \mathrm{~b}$ ). Further reductions were observed at 1 and 2 MED. The UV dose dependence for loss of type III procollagen and $\mathrm{pN}$ collagen was similar to that for type I procollagen (data not shown). Solar-simulated UV also caused significant reduction of type I procollagen and $\mathrm{pN}$ collagen (Figure $4 c)$. However, reduced procollagen levels were not observed at doses below 1 MED.

Involvement of c-Jun in UV inbibition of type I procollagen synthesis. The data presented above indicate that UV irradiation inhibits type I and type III procollagen gene expression in human skin in vivo. We next sought to gain some insight into the mechanism(s) responsible for this inhibition. Type I and type III procollagen genes are subject to both positive and negative regulation by a large number of different tran-
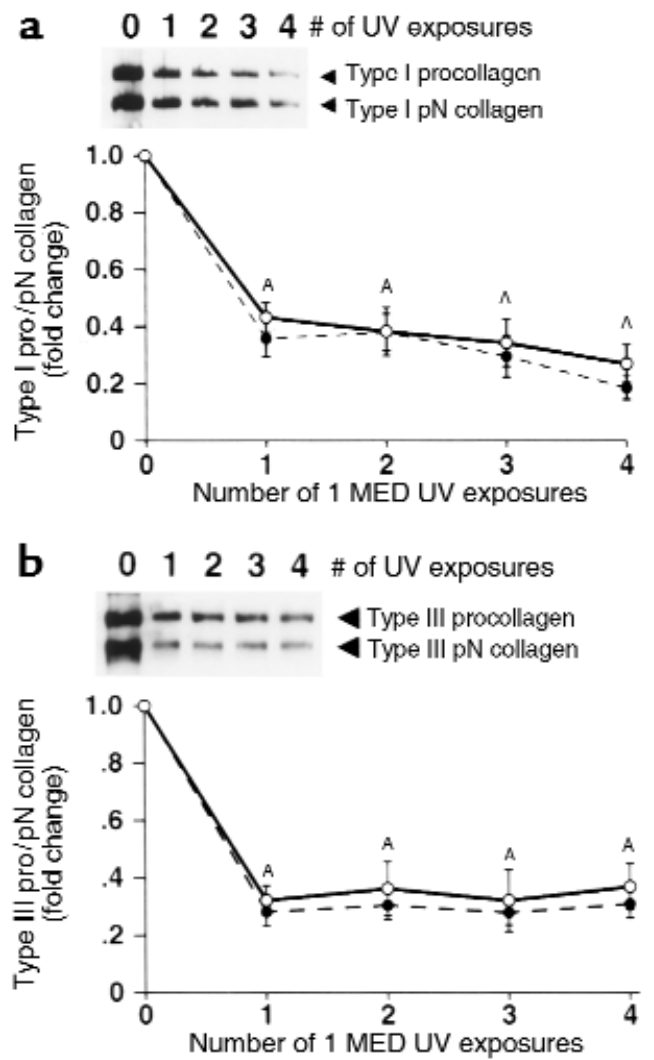

Figure 3

Multiple UV exposures cause sustained reduction of type I and type III procollagen proteins in human skin in vivo. Human skin was exposed to one, two, three, or four doses of UV (1 MED) at 24-hour intervals. Skin biopsies were obtained 24 hours after each exposure. Skin samples were analyzed for type $\mathrm{I}(\alpha 1)$ and type $\mathrm{III}(\alpha 1)$ procollagen and $\mathrm{pN}$ collagen protein levels using Western blot method. (a) Type I procollagen (open circle, solid line) and type I pN collagen (filled circle, broken line) protein in human skin. Inset shows representative Western blot. $n=7$ subjects. (b) Type III procollagen (open circle, solid line) and type III pN collagen (filled circle, broken line) protein in human skin. Inset shows representative Western blot. $n=6$ subjects. ${ }^{A} P<0.05$ vs. no UV exposure. 
a

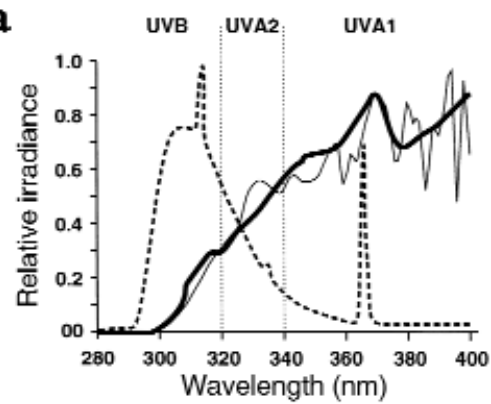

b NO

UV $0.10 .5 \quad 1 \quad 2$ MED

Type I procollagen

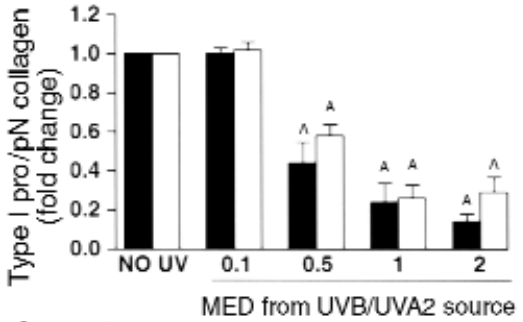

C NO

UV 0.10 .5112 MED

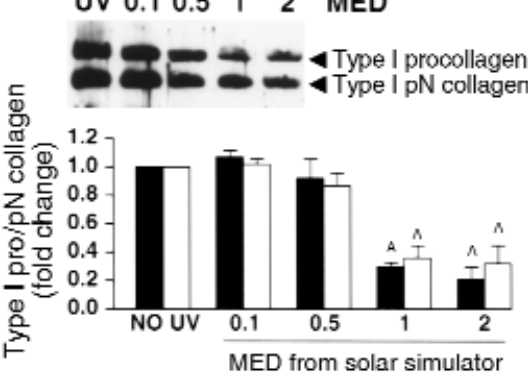

Figure 4

UVB/UVA2 and solar-simulated UV dose dependence for reduction of type I procollagen in human skin in vivo. Human skin was irradiated with the indicated doses of UV from either a UVB/UVA2 source or a solar simulator. Skin was obtained 24 hours after irradiation and analyzed for type I procollagen protein levels using the Western blot method. (a) UV spectra for Kodacel-filtered UVB/UVA2 source (broken line), solar simulator (thick solid line), and sunlight (thin solid line). (b) UVB/UVA2 dose dependence for reduction of type I procollagen (filled bars) and type I pN collagen (open bars) in human skin. Inset shows representative Western blot. $n=5$ subjects. (c) Solar-simulated UV dose dependence for reduction of type I procollagen (filled bars) and type I $\mathrm{pN}$ collagen (open bars) in human skin. Inset shows representative Western blot. $n=5$ subjects. ${ }^{A} P<0.05$ vs. no UV exposure.

scription factors (29-31). Despite this complexity, recent data indicate that a binding site within the promoter of the type $\mathrm{I}(\alpha 2)$ procollagen gene for transcription factor AP- 1 is required for regulated gene expression (32). This AP-1 site (located between -264 and -241) was shown to bind the AP-1 family member Jun $B$ and to be necessary for basal and TGF- $\beta$ induced expression of type $\mathrm{I}(\alpha 2)$ procollagen. Interestingly, TGF- $\beta$ induction of type $\mathrm{I}(\alpha 2)$ procollagen gene expression was inhibited by overexpression of cJun, another member of the AP-1 family (29).
We have reported previously that UV irradiation causes sustained induction (at least 24 hours) of c-Jun in human skin in vivo (21). We therefore investigated the role of c-Jun in the observed inhibition of procollagen gene expression by UV irradiation. For these studies we used primary adult human skin fibroblasts. UV irradiation of the fibroblasts caused a transient induction of cJun protein (Figure 5a). We found that c-Jun was induced (fivefold) within 4 hours, remained elevated for at least 8 hours, and returned to near baseline by 16 hours after UV irradiation. Human skin fibroblasts were transfected with a CAT reporter gene under the control of type $I(\alpha 2)$ procollagen proximal gene promoter $(-772$ to $+58 \mathrm{bp}$ ), containing the critical AP-1-binding site. This reporter was constitutively active in human fibroblasts (Figure 5b). Promoter activity was significantly reduced 8 hours after exposure of the fibroblasts to UV irradiation. Overexpression of wild-type c-Jun further suppressed procollagen $\mathrm{I}(\alpha 2)$ promoter activity in UV-irradiated fibroblasts. In contrast, overexpression of dominant-negative mutant c-Jun abrogated UV-induced
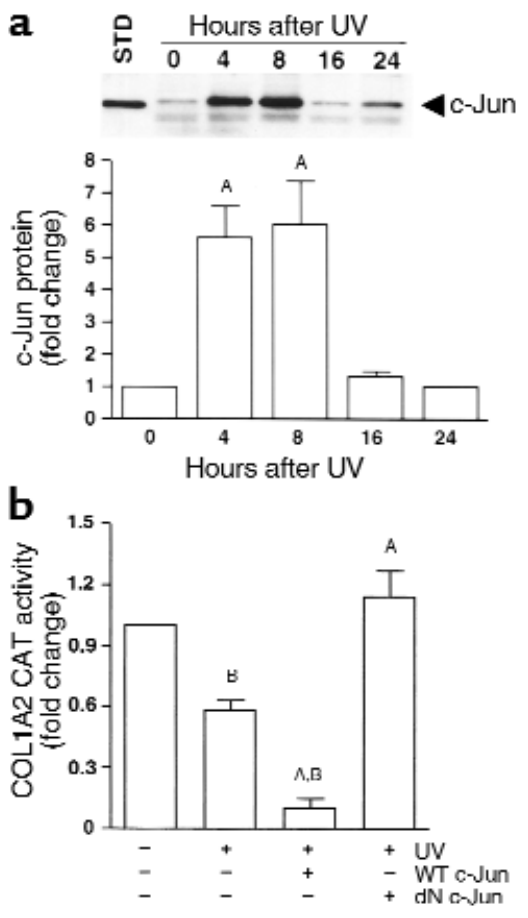

Figure 5

UV-induced c-Jun inhibits type $\mathrm{I}(\alpha 2)$ procollagen gene expression in primary human skin fibroblasts. (a) Time course of UV induction of c-Jun protein in human skin fibroblasts. Nuclear extracts were prepared from fibroblasts at the indicated times after UV irradiation (30 $\mathrm{mJ} / \mathrm{cm}^{2}$ ). The c-Jun protein levels were determined using the Western blot method. Inset shows a representative Western blot. $n=5$ subjects. ${ }^{A} P<0.5$ vs. no UV exposure. (b) c-Jun mediates UV inhibition of type $\mathrm{I}(\alpha 2)$ procollagen gene-promoter activity in human skin fibroblasts. Fibroblasts were cotransfected with type I( $\alpha 2)$ procollagen promoter CAT reporter alone or with either wild-type (WT) c-Jun or dominant-negative mutant $(\mathrm{dN}) \mathrm{c}$-Jun expression vectors, as indicated. CAT activity was determined 12 hours after irradiation $\left(30 \mathrm{~mJ} / \mathrm{cm}^{2}\right)$. $n=5$ subjects. ${ }^{A} P \leq 0.05$ vs. UV exposure; ${ }^{B} P \leq 0.05$ vs. control. 
a

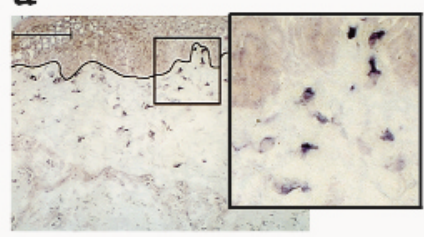

c

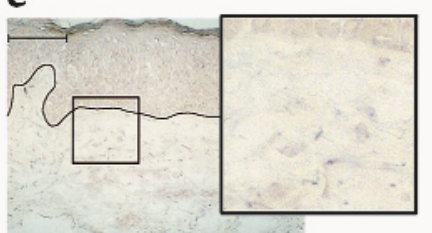

e

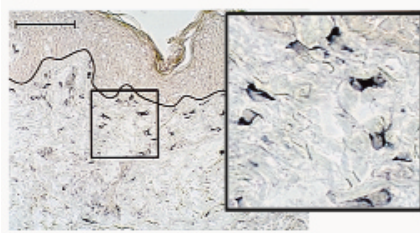

g

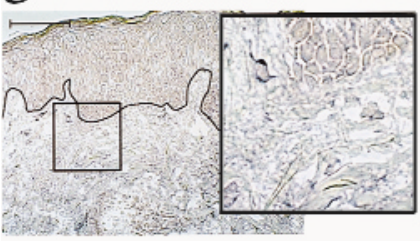

b



d

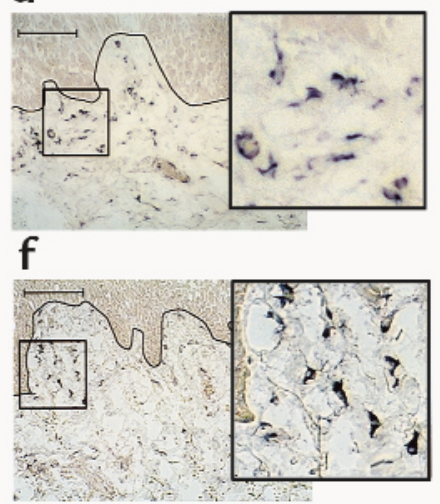

h

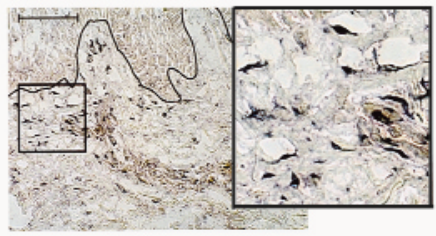

\section{Figure 6}

All-trans retinoic acid (RA) protects against UV-induced reduction of type I and type III procollagen mRNA expression in human skin in vivo. Human skin was pretreated with vehicle and $0.1 \%$ RA for 24 hours, then irradiated with UV ( 2 MED). Skin was obtained 24 hours after UV exposure. Type I (a-d) and type III (e-h) procollagen mRNA expression was determined by digoxigenin riboprobe in situ hybridization. $\mathbf{a}$ and $\mathbf{e}$, vehicle-treated skin; $\mathbf{b}$ and $\mathbf{f}, \mathrm{RA}$-treated skin; $\mathbf{c}$ and $\mathbf{g}$, vehicle-pretreated, UVirradiated skin; $\mathbf{d}$ and $\mathbf{h}$, RA-pretreated, UV-irradiated skin. Insets show enlargement of cells in the dermis. Solid black lines demarcate border between epidermis and connective tissue. Areas outlined in black are shown in 2.5fold enlargements. Data displayed are representative of six subjects. Scale bar $=10 \mu \mathrm{m}$.

tially protected against loss of type I and type III procollagen mRNA and protein. Treatment of skin with all-trans retinoic acid alone had no effect on type I or type III procollagen mRNA or protein levels.

\section{Discussion}

The data demonstrate that UV irradiation causes reduction of type I and type III procollagen mRNA and protein expression in fibroblasts in human skin in vivo. UV reduction of procollagen mRNA and protein

inhibition of type I procollagen promoter activity (Figure $5 b)$. Northern analyses revealed that UV irradiation reduced endogenous type $I(\alpha 1)$, type $I(\alpha 2)$, and type $\mathrm{III}(\alpha 1)$ procollagen gene expression in cultured human skin fibroblasts. Eight hours after UV irradiation, type $\mathrm{I}(\alpha 1)$, type $\mathrm{I}(\alpha 2)$, and type III $(\alpha 1)$ procollagen mRNA levels were reduced $37 \pm 9 \%(n=5, P<0.01) ; 52 \pm 5 \%(n=8$, $P<0.01)$; and $46 \pm 6 \%(n=3, P<0.01)$, respectively. These data support the involvement of UV-induced c-Jun in UV inhibition of type $\mathrm{I}(\alpha 2)$ procollagen gene expression.

All-trans retinoic acid protects against $U V$-induced inbibition of procollagen synthesis in human skin in vivo. Pretreatment of skin with all-trans retinoic acid inhibits accumulation of c-Jun protein in UV-irradiated human skin (21). The data presented implicate c-Jun induction in UV-irradiation inhibition of procollagen synthesis. We therefore investigated whether pretreatment of human skin with all-trans retinoic acid could protect against UV-induced inhibition of type I and type III procollagen synthesis. Human skin was treated with vehicle or all-trans retinoic acid (0.1\%) 24 hours before UV irradiation (2 MED). Skin samples were obtained 24 hours after irradiation and analyzed for type I $(\alpha 1)$ and type III $(\alpha 1)$ procollagen gene expression by in situ hybridization, and protein levels were analyzed using the Western blot method. As expected, type I and type III procollagen mRNA (Figure 6) and protein (Figure 7) expression were significantly reduced in vehicle-treated, UV-irradiated (compared with vehicle-treated, nonirradiated) human skin. Pretreatment of skin with all-trans retinoic acid substan- expression was most prominent in the upper one-third of skin connective tissue. This localization likely reflects the depth of UV penetration into the skin, which increases with increasing wavelength. We used a UVB/UVA2 source that emits wavelengths primarily between 290-340 nm, which penetrates into the upper region of the connective tissue (33).

In nonirradiated human skin, type I and III procollagen proteins are present within fibroblasts and extracellularly. In the upper dermis, this procollagen pool is substantially reduced within 8 hours and essentially absent within 24 hours after UV irradiation. This depletion of existing procollagen protein presumably reflects ongoing conversion into mature collagen coupled with decreased new procollagen synthesis due to reduced procollagen mRNA levels. However, our data do not allow us to directly ascertain the fate of the existing procollagen protein pool. It is possible that UV irradiation stimulates degradation and/or processing of procollagen.

We have reported previously that UV irradiation induces collagenase and other matrix metalloproteinases in human skin in vivo $(19,20)$. Collagenase is induced 12-16 hours after UV irradiation, trailing the initial loss of procollagen protein, which is observed 8 hours after UV irradiation. This difference in the time of onset of collagenase induction and reduction of procollagen protein makes it unlikely that initial loss of procollagen results from UV-induced, collagenase-mediated degradation. In general, however, the time course for UV induction of matrix-metalloproteinase mRNA and pro- 
tein expression inversely mirrors that for UV reduction of procollagen mRNA and protein expression. During the first 24 hours after UV exposure, matrix-metalloproteinase mRNAs and proteins are induced, whereas procollagen mRNAs and proteins are reduced. During the second and third days after UV exposure, matrixmetalloproteinase expression declines, whereas procollagen expression increases. Multiple UV exposures cause sustained elevations of matrix-metalloproteinase mRNAs and proteins (19) and sustained repression of procollagen mRNAs and proteins. Simultaneous reduction of procollagen expression and induction of mature collagen degradation maximizes mature collagen loss during the initial 24 hours after a single UV exposure. During the succeeding 48 hours (i.e., 2-3 days after UV exposure) matrix-metalloproteinase expression wanes as procollagen expression rises. This temporal coordination of matrix metalloproteinase-mediated collagen breakdown and procollagen mRNA and protein expression protects newly synthesized mature collagen (and procollagen) from rapid degradation.

Evidence suggests that transcriptional control is the major mechanism of regulation of type I procollagen expression $(26,29,34-37)$. Transcription factor AP-1, which is composed of Jun and Fos proteins, negatively regulates both type $\mathrm{I}(\alpha 1)$ and type $\mathrm{I}(\alpha 2)$ procollagen gene transcription $(31,32,37)$. UV irradiation rapidly upregulates AP-1 in human skin in vivo. This induction of AP-1 results primarily from increased c-Jun expression, since c-Fos is constitutively expressed in human skin $(20,21)$. c-Jun remains maximally elevated for at least 24 hours after UV irradiation in human skin in vivo. This sustained induction of c-Jun is consistent with the observed time course for repression of procollagen gene expression. These observations led us to investigate the role of c-Jun in UV inhibition of type I procollagen gene expression in primary adult human skin fibroblasts. UV irradiation of skin fibroblasts rapidly induced c-Jun and inhibited endogenous type I and type III procollagen gene expression. We found that human type $\mathrm{I}(\alpha 1)$ procollagen-promoter CAT-reporter constructs, which were previously characterized in mouse 3T3 fibroblasts (29), were not transcriptionally active in adult human skin fibroblasts and therefore could not be studied. In contrast, the human type I $(\alpha 2)$ procollagen promoter CAT reporter (containing $714 \mathrm{bp}$ upstream from the transcription start site) was active in human skin fibroblasts. Promoter activity was reduced by UV irradiation and further reduced by overexpression of wild-type c-Jun. Overexpression of dominantnegative mutant c-Jun completely abrogated UV inhibition of promoter activity. In cultured fibroblasts, c-Jun remained elevated for at least 8 hours after UV irradiation (as opposed to 24 hours in human skin in vivo). In cultured fibroblasts, UV inhibition of endogenous type $I(\alpha 2)$ procollagen gene expression and type $\mathrm{I}(\alpha 2)$ procollagen promoter reporter was maximal 8 hours after UV exposure. Therefore, the kinetics of induction of c-Jun and repression of type $\mathrm{I}(\alpha 2)$ procol- lagen promoter activity coincided. These data indicate that UV inhibition of type $\mathrm{I}(\alpha 2)$ procollagen gene expression in adult human fibroblasts is mediated, at least in part, by UV-induced c-Jun. However, these data do not rule out the possibility that there may be other factors, including reduced mRNA stability, that contribute to UV inhibition of procollagen gene expression.

Treatment of human skin in vivo with all-trans retinoic acid before UV irradiation protected against UV-induced loss of type I and type III procollagen transcripts and proteins. Protection by all-trans retinoic acid was observed after 24 hours of pretreatment, but not after 8 hours of pretreatment (data not shown). We have shown previously that pretreatment of human skin with all-trans retinoic acid for 24 hours, but not 8 hours, inhibits induction of c-Jun and AP-1 (19-21). These data support the involvement of c-Jun in UV inhibition of type I and type III procollagen expression
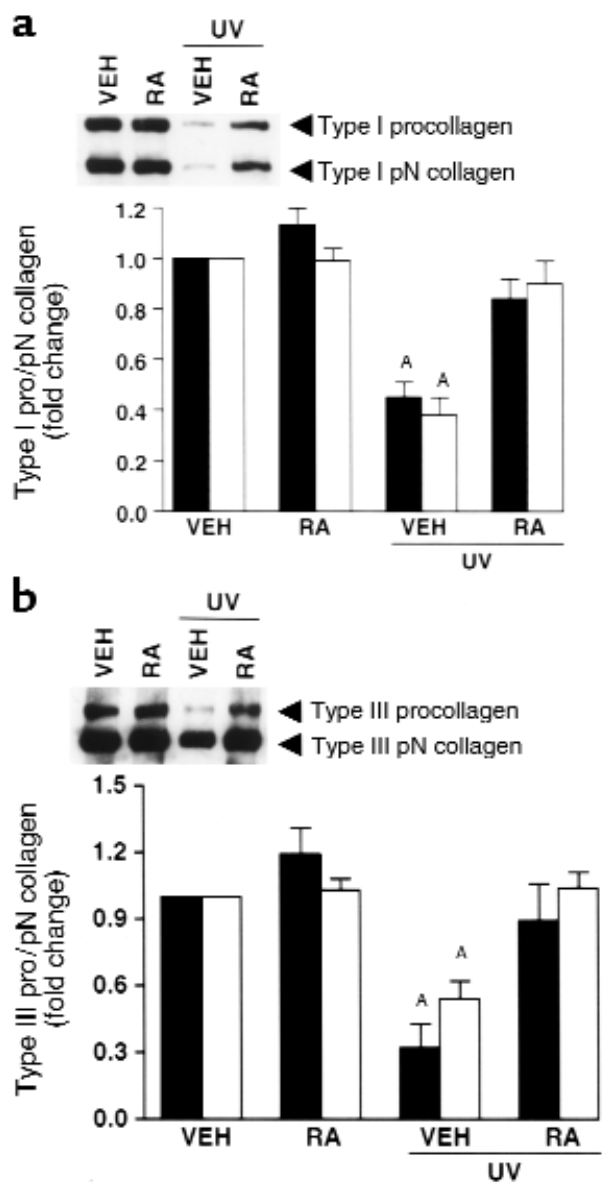

\section{Figure 7}

All-trans retinoic acid (RA) protects against UV-induced loss of type I and type III procollagen proteins in human skin in vivo. Human skin was pretreated with vehicle (VEH) and $0.1 \%$ RA for 24 hours, then irradiated with UV (2 MED). Skin was obtained 24 hours after UV exposure. (a) Type I procollagen (filled bars) and type I pN collagen (open bars) protein in human skin. Inset shows representative Western blot. (b) Type III procollagen (filled bars) and type III pN collagen (open bars) protein in human skin. Inset shows representative Western blot. $n=9$ subjects. ${ }^{A} P<0.05$ vs. vehicle-treated, nonirradiated skin. 
in human skin in vivo. The requirement for prolonged pretreatment with all-trans retinoic acid presumably reflects a mechanism that involves new gene expression, however, this mechanism remains to be clarified.

Type I procollagen and type III procollagen are reduced in chronically photodamaged human skin, not recently exposed to UV $(5,6,11)$. Treatment of such photodamaged skin with all-trans retinoic acid increases type I procollagen expression (11). The mechanism of this increase is not known, however, it may involve increased expression or activation of TGF- $\beta(38,39)$. In addition, treatment of skin with all-trans retinoic acid before UV exposure inhibits collagen breakdown by matrix metalloproteinases $(19,20)$ and protects against UVinduced reduction of procollagen expression, as demonstrated in this study. Therefore, all-trans retinoic acid and its metabolic precursor all-trans retinol (vitamin A) should have the capacity to both repair existing photodamage and retard accumulation of new photodamage.

\section{Acknowledgments}

The authors would like to acknowledge Carolyn Petersen and Suzan Rehbine for tissue procurement, Laura VanGoor for graphics preparation, Ted Hamilton for statistical analyses, and Anne Chapple for editorial assistance. We thank Maria Trojanowska at Medical University of South Carolina for COL1A2 construct.

This study was supported in part by the Babcock Endowment for Dermatological Research and a grant from the Johnson \& Johnson Corporation.

1. Kligman, A.M. 1969. Early destructive effects of sunlight on human skin. JAMA. 210:2377-2380.

2. Bernstein, E.F., et al. 1996. Long-term sun exposure alters the collagen of the papillary dermis: comparison of sun-protected and photoaged skin by Northern analysis, immunohistochemical staining, and confocal laser scanning microscopy. J. Am. Acad. Dermatol. 34:209-218.

3. Lavker, R.M. 1995. Cutaneous aging: chronologic versus photoaging. In Photoaging. B.A. Gilchrest, editor. Blackwell Science. Cambridge, Massachusetts, USA. 123-135.

4. Uitto, J. 1993. Collagen. In Dermatology in general medicine. Volume 1. T.B. Fitzpatrick, A.Z. Eisen, K. Wolff, I.M. Freedberg, and K.F. Austen, editors. McGraw-Hill. New York, New York, USA. 299-314.

5. Talwar, H.S., Griffiths, C.E.M., Fisher, G.J., Hamilton, T.A., and Voorhees, J.J. 1995. Reduced type I and type III procollagens in photodamaged adult human skin. J. Invest. Dermatol. 105:285-291.

6. Schwartz, E., Cruickshank, F.A., Christensen, C.C., Perlish, J.S., and Lebwohl, M. 1993. Collagen alterations in chronically sun-damaged human skin. Photochem. Photobiol. 58:841-844.

7. Yamauchi, M., Prisayanh, P., Haque, Z., and Woodley, D.T. 1991. Collagen cross-linking in sun-exposed and unexposed sites of aged human skin. J. Invest. Dermatol. 97:938-941.

8. Calderone, D.C., and Fenske, N.A. 1995. The clinical spectrum of actinic elastosis. J. Am. Acad. Dermatol. 32:1014-1024.

9. Bryce, G.F., Bogdan, N.J., and Brown, C.C. 1988. Retinoic acids promote the repair of the dermal damage and the effacement of wrinkles in the UVB-irradiated hairless mouse. J. Invest. Dermatol. 91:175-180.

10. Chen, S., Kiss, I., and Tramposch, K.M. 1992. Effects of all-trans retinoic acid on UVB-irradiated and non-irradiated hairless mouse skin. J. Invest. Dermatol. 98:248-254.

11. Griffiths, C.E.M., et al. 1993. Restoration of collagen formation in photodamaged human skin by tretinoin (retinoic acid). N. Engl. J. Med. 329:530-535.

12. Uitto, J. 1979. Collagen polymorphism: isolation and partial characterization of alpha 1(I)-trimer molecules in normal human skin. Arch. Biochem. Biophys. 192:371-379.

13. Deak, S.B.M., Pope, F.M., and Prockop, D.J. 1983. The molecular defect in a nonlethal variant of osteogenesis imperfecta. J. Biol. Chem. 258:15192-15197.
14. Halila, R., and Peltonen, L. 1986. Purification of human procollagen type III N-proteinase from placenta and preparation of antiserum. Biochem.J. 239:47-52.

15. Hojima, Y., McKenzie, J.A., Van der Rest, M., and Prockop, D.J. 1989. Type I procollagen $\mathrm{N}$ proteinase from chick embryo tendons. Purification of a new $500-\mathrm{kDa}$ form of the enzyme and identification of the catalytically active polypeptides. J. Biol. Chem. 264:11336-11345.

16. Oikarinen, A., Autio, P., Kiistala, U., Risteli, L., and Risteli, J. 1992. A new method to measure type I and III collagen synthesis in human skin in vivo: demonstration of decreased collagen synthesis after topical glucocorticoid treatment. J. Invest. Dermatol. 98:220-225.

17. Haukipuro, K., Meikko, J., Risteli, L., Kairaluoma, M.I., and Risteli, J. 1991. Synthesis of Type I collagen in healing wounds in humans. Ann. Surg. 213:75-80.

18. Liu, X., et al. 1995. A targeted mutation at the known collagenase cleavage site in mouse type I collagen impairs tissue remodeling. J. Cell. Biol. 130:227-237.

19. Fisher, G.J., et al. 1997. Pathophysiology of premature skin aging induced by ultraviolet light. N. Engl. J. Med. 337:1419-1428.

20. Fisher, G.J., et al. 1996. Molecular basis of sun-induced premature skin ageing and retinoid antagonism. Nature. 379:335-339.

21. Fisher, G.J., et al. 1998. Retinoic acid inhibits induction of c-Jun protein by ultraviolet irradiation that occurs subsequent to activation of mitogen-activated protein kinase pathways in human skin in vivo. J. Clin. Invest. 101:1432-1440.

22. Fisher, G.J., Esmann, J., Griffiths, C.E.M., and Voorhees, J.J. 1991. Cellular, immunologic and biochemical characterization of topical retinoic acid-treated human skin. J. Invest. Dermatol. 96:699-707.

23. Fisher, G.J., et al. 1994. Immunological identification and functional quantitation of retinoic acid and retinoid $X$ receptor proteins in human skin. J. Biol. Chem. 269:20629-20635.

24. Fisher, G.J., et al. 1995. All-trans retinoic acid induces cellular retinolbinding protein in human skin in vivo. J. Invest. Dermatol. 105:80-86.

25. Reynolds, N.J., et al. 1993. Differential induction of phosphatidylcholine hydrolysis, diacylglycerol formation and protein kinase $\mathrm{C}$ activation by epidermal growth factor and transforming growth factor- $\alpha$ in normal human skin fibroblasts and keratinocytes. Biochem. J. 294:535-544.

26. Ihn, H., LeRoy, E.C., and Trojanowska, M. 1997. Oncostatin M stimulates transcription of the human $\alpha 2$ (I) collagen gene via the Sp1/Sp3binding site. J. Biol. Chem. 272:24666-24672.

27. Brown, P.H., Chen, T.K., and Birrer, M.J. 1994. Mechanism of action of a dominant-negative mutant of c-Jun. Oncogene. 9:791-799.

28. Sambrook, J., Fritsch, E.F., and Maniatis, T. 1989. Molecular cloning: a laboratory manual. 2nd edition, volume 3. Cold Spring Harbor Laboratory Press. Cold Spring Harbor, New York, USA. p. 16.59.

29. Jimenez, S.A., et al. 1994. Functional analysis of human $\alpha 1$ (I) procollagen gene promoter. J. Biol. Chem. 269:12684-12691.

30. Ihn, H., Ohnishi, K., Tamaki, T., LeRoy, E.C., and Trojanowski, M. 1996. Transcriptional regulation of the human $\alpha 2(\mathrm{I})$ collagen gene. J. Biol. Chem. 271:26717-26723.

31. Bornstein, P. 1996. Regulation of expression of the $\alpha 1$ (I) collagen gene: a critical appraisal of the role of the first intron. Matrix Biol. 15:3-10.

32. Chung, K., Agarwal, A., Uitto, J., and Mauviel, A. 1996. An AP-1 binding sequence is essential for regulation of the human $\alpha 2(\mathrm{I})$ collagen (COL1A2) promoter activity by transforming growth factor- $\beta$. J. Biol. Chem. 271:3272-3278.

33. Shea, R.C., and Parish, J.A. 1991. Nonionizing radiation and the skin. In Physiology, biochemistry, and molecular biology of the skin. Volume 2. L.A. Goldsmith, editor. Oxford University Press. New York, New York, USA. 910-927.

34. Rossert, J.A., and Garrett, L.A. 1995. Regulation of type I collagen synthesis. Kidney Int. 47:1421-1432.

35. Inagaki, Y., Truter, S., Tanaka, S., Di Liberto, M., and Ramirez, F. 1995. Overlapping pathways mediate the opposing actions of tumor necrosis factor- $\alpha$ and transforming growth factor- $\beta$ on $\alpha 2$ (I) collagen gene transcription. J. Biol. Chem. 270:3353-3358.

36. Tamaki, T., Ohnishi, K., Hartl, C., LeRoy, E.C., and Trojanowska, M. 1995. Characterization of a GC-rich region containing Sp1 binding sites as a constitutive responsive element of the $\alpha 2$ (I) collagen gene in human fibroblasts. J. Biol. Chem. 270:4299-4304.

37. Philips, N., Bashey, R.I., and Jimenez, S.A. 1995. Increased $\alpha 1(\mathrm{I})$ procollagen gene expression in tight skin (TSK) mice myocardial fibroblasts is due to a reduced interaction of a negative regulatory sequence with AP1 transcription factor. J. Biol. Chem. 270:9313-9321.

38. Glick, A.B., Flanders, K.C., Danielpour, D., Yuspa, S.H., and Sporn, M.B. 1989. Retinoic acid induces transforming growth factor-beta 2 in cultured keratinocytes and mouse epidermis. Cell Regul. 1:87-97.

39. Fisher, G., et al. 1992. Differential modulation of transforming growth factor- $\beta 1$ expression and mucin deposition by retinoic acid and sodium lauryl sulfate in human skin. J. Invest. Dermatol. 98:102-108. 